\title{
Threat Prediction from Schemas as a Source of Bias in Pain Perception
}

\author{
Manyoel Lim, ${ }^{1,2}$ Christopher 0'Grady, ${ }^{1,2}$ Douglas Cane, ${ }^{2}$ Amita Goyal, ${ }^{2}$ Mary Lynch, ${ }^{1,2}$ Steven Beyea, ${ }^{3}$ \\ and Javeria Ali Hashmi ${ }^{1,2}$ \\ ${ }^{1}$ Department of Anesthesia, Pain Management and Perioperative Medicine, Dalhousie University, Halifax, Nova Scotia B3H 4R2, Canada, ${ }^{2}$ Nova Scotia \\ Health Authority, Halifax, Nova Scotia B3H 1V7, Canada, and ${ }^{3}$ Biomedical Translational Imaging Centre (BIOTIC), QEII Health Sciences Centre, and \\ Department of Diagnostic Radiology, Dalhousie University, Halifax, Nova Scotia, B3H 3A7, Canada
}

Our sensory impressions of pain are generally thought to represent the noxious properties of an agent but can be influenced by the predicted level of threat. Predictions can be sourced from higher-order cognitive processes, such as schemas, but the extent to which schemas can influence pain perception relative to bottom-up sensory inputs and the underlying neural underpinnings of such a phenomenon are unclear. Here, we investigate how threat predictions generated from learning a cognitive schema lead to inaccurate sensory impressions of the pain stimulus. Healthy male and female participants first detected a linear association between cue values and stimulus intensity and rated pain to reflect the linear schema when compared with uncued heat stimuli. The effect of bias on pain ratings was reduced when prediction errors (PEs) increased, but pain perception was only partially updated when measured against stepped increases in PEs. Cognitive, striatal, and sensory regions graded their responses to changes in predicted threat despite the PEs $(p<0.05$, corrected). Individuals with more catastrophic thinking about pain and with low mindfulness were significantly more reliant on the schema than on the sensory evidence from the pain stimulus. These behavioral differences mapped to variability in responses of the striatum and ventromedial prefrontal cortex. Thus, this study demonstrates a significant role of higher-order schemas in pain perception and indicates that pain perception is biased more toward predictions and less toward nociceptive inputs in individuals who report less mindfulness and more fear of pain.

Key words: prediction; expectation; pain; fMRI; cognitive; bias

\section{Significance Statement}

This study demonstrates that threat predictions generated from cognitive schemas continue to influence pain perception despite increasing prediction errors arising in pain pathways. Individuals first formed a cognitive schema of linearity in the relationship between the cued threat value and the stimulus intensity. Subsequently, the linearity was reduced gradually, and participants partially updated their evaluations of pain in relation to the stepped increases in prediction errors. Individuals who continued to rate pain based more on the predicted threat than on changes in nociceptive inputs reported high pain catastrophizing and less mindful-awareness scores. These two affects mapped to activity in the ventral and dorsal striatum, respectively. These findings direct us to a significant role of top-down processes in pain perception.

\section{Introduction}

Pain is necessary for survival because it signals physical threats and helps to reinforce aversive behaviors toward noxious stimuli

Received Sept. 2, 2019; revised Nov. 1, 2019; accepted Dec. 3, 2019.

Author contributions: S.B. and J.A.H. designed research; M. Lim, C.O., D.C., A.G., M. Lynch, S.B., and J.A.H. performed research; M. Lim, M. Lynch, S.B., and J.A.H. contributed unpublished reagents/analytic tools; M. Lim and J.A.H. analyzed data; M. Lim, C.O., D.C., A.G., S.B., and J.A.H. wrote the paper

This research was supported by a Natural Sciences and Engineering Research Council of Canada Discovery Grant, Canada Research Chairs Program, Canadian Foundation for Innovation Grant, Nova Scotia Health Authority (NSHA) Establishment Grant, NSHA Fibromyalgia Grant, Dalhousie start-up funds to J.A.H., Brain Canada Grant, Radiology Research Fund to S.B., and Basic Science Research Program through the National Research Foundation of Korea (Grant 2017R1A6A3A03003741) to M. Lim.

The authors declare no competing financial interests.

Correspondence should be addressed to Javeria Ali Hashmi at javeria.hashmi@dal.ca.
(Baliki et al., 2010; Zubieta, 2010; Seymour, 2019). Pain signals are not only necessary for instantly responding to physical threats but are also important for predicting the inherent harm in objects or situations (Loeser and Treede, 2008; Wang et al., 2018). By gauging pain intensity, our sensory systems learn how to appropriately infer potential harm and can generate appropriate approach-withdraw decisions.

A prediction is a representation about a future event built on prior experience and knowledge. Also referred to as expectations, predictions are generated from memory and cued associations 
(O'Doherty et al., 2017) and are of a higher order when generated from abstracting associations based on logical rules (Wacongne et al., 2011; Russek et al., 2017). In natural environments, where sensory inputs are varied and uncertain, predictions are often derived from information patterns that are hierarchical and complex rather than simple or pavlovian (Egner and Summerfield, 2013; LeDoux and Daw, 2018). Mental constructs, referred to here as cognitive schemas, can be defined as superordinate knowledge built from multiple associations and cognitive reasoning (LeDoux and Daw, 2018). Schemas are higher-level knowledge structures, such as ideas and concepts. A useful adaptation for conserving resources, schemas allow us to rapidly extract meaning from sensory inputs. Studies have shown the influence of prior context on pain primarily with pavlovian conditioning and placebo suggestion (Ashar et al., 2017; Hashmi, 2018), but models that can test effects of cognitive processes such as logical reasoning in building top-down schemas of the relationship between cue and pain properties have not yet been tested. Thus, the direct influence of higher-order predictions in directing pain perception remains unclear.

Understanding how the brain responds when higher-order predictions are incorrect is pertinent for understanding perceptual biases. When sensory evidence fails to confirm the prediction, either the sensory evidence is ignored and, in turn, biases perception, or the brain learns from the error and updates subsequent predictions and evaluations to keep bias in check (Feldman and Friston, 2010; Rigoli et al., 2016). For higher-order predictions, such as those based on an internal logic or a priori mathematical reasoning, the influence on perceived pain may be even more tenacious (Friston and Kiebel, 2009; LeDoux and Daw, 2018; Weilnhammer et al., 2018). Previous studies have demonstrated that the effect of expectations on pain is not fully extinguished, even when prediction errors (PEs) of the same magnitude are repeated (Schenk et al., 2017; Zunhammer et al., 2018), but the effect of schema-induced bias and increases in prediction errors has not yet been systematically tested.

Identifying brain systems that mediate effects of predictions from threat schemas is necessary for understanding why people perceive pain and respond to physical threats differently (Garrett et al., 2018). Some individuals are more sensitive to predicted threats (Karos et al., 2018), resulting in distorted pain experiences (Boichat et al., 2018) and corresponding to a higher self-report of pain catastrophizing (Elman and Borsook, 2018). In contrast, higher bodily awareness (mindfulness) is said to be useful for reducing the influence of maladaptive thought processes and can relieve persistent pains (Harrison et al., 2019). Although mindfulness and catastrophizing are inversely linked, these two factors may affect pain perception by altering predictive processes in the brain.

Here, we investigate how pain ratings will deviate from a linear schema when sensory evidence from bottom-up signals deviates from a schema at increasing levels of prediction errors. The neuroimaging analysis was targeted at determining the extent to which top-down cognitive and bottom-up sensory systems modulate their response, with changes in cued threat values and prediction errors. In addition, we measured the influence of catastrophizing and mindfulness as factors that mediate perceptual bias. We hypothesized that pain ratings deviate from the linear schema when prediction errors from bottom-up pain pathways increase, but the schema continues to influence pain ratings even when the prediction error is at a maximum (max).

\section{Materials and Methods}

Study participants. Forty-two healthy participants (21 women; mean \pm SD age, $31.23 \pm 10.91$ years; age range, $20-56$ years) participated in the study. Inclusion criteria for healthy participants were as follows: (1) between 18 and 75 years of age, and (2) right handed. Participants were excluded if they (1) had concomitant acute or chronic pain; (2) were taking medications for pain; (3) were pregnant; (4) had a history of cardiac, respiratory, or nervous system disease that may interfere with participation in the study or potential for adverse outcome (e.g., asthma or psychiatric or mental disorders); or (5) had contraindications to MRI scanning [e.g., cardiac pacemaker, metal implants (including titanium), dental braces, permanent retainers, or known fear of closed spaces]. The study protocol was approved by the Nova Scotia Health Authority Research Ethics Board. All participants provided written informed consent before participation in the study. Analysis of behavioral data was conducted based on all 42 participants, but complete fMRI data could not be collected for four participants and were excluded from analysis.

Thermal pain stimulation. Painful heat stimuli were delivered to the skin on the left lower leg (tibialis anterior muscle) using an ATS $30 \times 30$ $\mathrm{mm}$ thermode (PATHWAY system, Medoc). Before the MRI scan, heat pain thresholds and tolerance were measured through an ascending heat stimulus using the method of limits. The temperature was slowly increased from a neutral baseline $\left(35^{\circ} \mathrm{C}\right)$ at a rate of $1 \%$ s. The participant pressed a button to terminate the stimulus as soon as they detected pain to measure pain threshold or as soon as the evoked pain was intolerable to measure pain tolerance. All thresholds were measured three times and averaged for further analysis. During the fMRI scan, the stimulation started at a baseline temperature of $35^{\circ} \mathrm{C}$. The temperature change rate both from the baseline to destination and from destination back to baseline was $4^{\circ} \mathrm{C} / \mathrm{s}$. Each stimulus was presented for $8 \mathrm{~s}$, including the period of temperature change. In this study, we defined a temperature of $45^{\circ} \mathrm{C}$ as a low-intensity stimulus and a temperature of $47^{\circ} \mathrm{C}$ as a high-intensity stimulus. Plateau durations for high-intensity $\left(47^{\circ} \mathrm{C}\right)$ and low-intensity $\left(45^{\circ} \mathrm{C}\right)$ stimuli were 2 and $3 \mathrm{~s}$, respectively. After heat stimulation, participants rated perceived pain based on a $0-100$ numerical rating scale (NRS; $0=$ no pain as the left visual anchor points, $100=$ worst pain imaginable as the right visual anchor points). Participants moved the cursor on the scale to select a number representing their pain by pressing two buttons on an MRI-compatible response pad (Lumina LSC-400 controller, Cedrus) under their right hands (Fig. 1A).

Experimental task. This model was developed as a cognitive test of bias to evaluate the influence of predictions relative to new sensory evidence at both a group and an individual level. This task measures the influence of change in bottom-up sensory evidence in countering the effects of predictions from the schema on pain ratings at increasing ranges of prediction errors (Fig. 1C). Instead of the commonly used pavlovian cues and explicit conditioning (Atlas and Wager, 2012; Roy et al., 2014; Wiech et al., 2014; Geuter et al., 2017; Tinnermann et al., 2017; Wang et al., 2018), participants detected the implicit linear association where cue values matched the stimuli, without explicit instructions (Fig. 1A,B). The cues stated the intensity of incoming stimuli with a number, where every change in value created anticipatory states of corresponding change in the threat of experiencing intense pain. To establish the effects of learning the schema on perceived pain, pain ratings and neural activation in response to uncued stimuli (Fig. 1D) were measured at baseline and compared with response to cue-matched stimuli (Fig. 1B). Prediction errors were added by altering stimulus ratings at increasing ranges of prediction errors, and any update in pain ratings was quantified.

Task details. Participants were told that they would be shown information on a screen, experience heat pain, and be asked to rate the experienced pain intensity on a scale. Participants were familiarized with visual and heat stimuli and trained to provide pain ratings.

Experimental conditions were distributed over four runs containing epochs consisting of presentations of a visual cue, heat pain stimuli, and pain-rating events. Thus, in each epoch, the duration of the visual cue was $4.75 \mathrm{~s}$ (Fig. 1A). After a delay of $1.9 \mathrm{~s}$ (black screen with white fixation cross), the heat pain stimulus was administered while participants viewed a red fixation cross. The duration of the heat stimulus was $8 \mathrm{~s}$. After a 
A

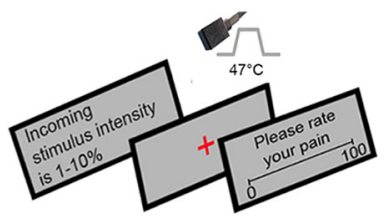

B

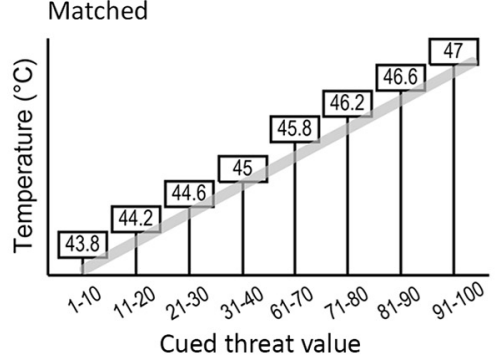

C

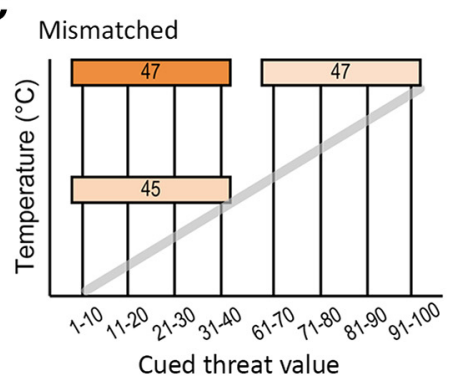

D

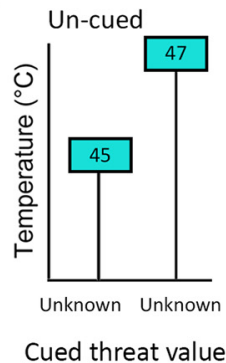

Figure 1. Schematic representation of the experimental paradigm. $A$, In each epoch, predictive cues were shown before a heat pain stimulus followed by a prompt for a pain rating on a $0-100$ numerical rating scale. The tested condition changed from epoch to epoch, and the order of the cued threat values and heat stimulus temperature was changed in a pseudorandom order. $\boldsymbol{B}$, Matched condition: the predictive cue values drawn from numbers between 1 and 100 , where each 10-point increase in cue value was associated with a $0.4^{\circ}$ increase in stimulus temperature as shown. Thus, predictive cue values increased in direct relation with the stimulus intensity. $\boldsymbol{C}$, Mismatched condition: PEs (difference between expected and actual temperatures) were introduced in two steps. In the first series of trials (Mismatched Level 1), cues ranging between 1 and 40 were paired with $45^{\circ} \mathrm{C}$, and those between 61 and 100 were paired with $47^{\circ} \mathrm{C}$, resulting in a range of PEs between $0^{\circ} \mathrm{C}$ and $1.2^{\circ} \mathrm{C}$. In the second series (Mismatched Level 2), a higher range of prediction errors was added by pairing the lower range of cues $(1-40)$ with $47^{\circ} \mathrm{C}$, resulting in a range of PEs between 0 and $3.2^{\circ} \mathrm{C}$ for the $47^{\circ} \mathrm{C}$ stimuli. $\boldsymbol{B}, \boldsymbol{C}$, The gray lines represent the expected response curve for the matched condition as a reference to the linear schema. $D$, Responses to $45^{\circ} \mathrm{C}$ and $47^{\circ} \mathrm{C}$ heat stimuli were quantified before the experiment and during the mismatched runs. Thus, no cue value was shown, and the visual cue indicated that the stimulus intensity is unknown.

delay of $4.75 \mathrm{~s}$ (black screen with white fixation cross), the NRS was displayed on the screen for $6 \mathrm{~s}$, and participants rated the intensity of experienced pain by moving a cursor along the scale. The interepoch interval ranged from 2.85 to $8.55 \mathrm{~s}$. Before the MRI scan, participants filled out questionnaires, and their responses to low-intensity $\left(45^{\circ} \mathrm{C}\right)$ and high-intensity $\left(47^{\circ} \mathrm{C}\right)$ stimuli were quantified at baseline to test how participants evaluated the pain stimuli when the cues did not contain information about stimulus intensity. Either $45^{\circ} \mathrm{C}$ or $47^{\circ} \mathrm{C}$ heat stimuli were delivered to participants, but the visual cues stated that "the incoming heat stimulus is at $x \%$ intensity." Stimuli $\left(45^{\circ} \mathrm{C}\right.$ and $\left.47^{\circ} \mathrm{C}\right)$ with no cue value were repeated three times.

All participants underwent structural and resting-state fMRI scans first, followed by the pain tasks with fMRI acquisitions. Task runs consisted of one matched condition run (Fig. 1B), one Mismatched Level 1 condition run, and two Mismatched Level 2 condition runs (Fig. 1C). In the Matched condition, the stimulus temperature was linearly increased with the cued threat values. The visual cues stated that "the incoming heat stimulus is at $x \%$ intensity." The value of $x$ varied between 1 and 100 with increments of 10 points (e.g., 1-10\%, 31-40\%). Each 10-point increase in cued threat value was associated with a $0.4^{\circ}$ increase in stimulus temperature (temperature range, $43.8-47^{\circ} \mathrm{C}$ ). Thus, during the matched condition, participants learned to predict the pain intensity from the cued threat value. The order of each epoch was pseudorandomized, and a total of 24 epochs were presented.

The remaining of three runs consisted of trials containing prediction errors (Fig. 1C). Thus, after the first matched condition run, in the second run (Mismatched Level 1 condition), cue values selected from a range of $1-40 \%$ were coupled with low-heat $\left(45^{\circ} \mathrm{C}\right)$ stimuli, and cue values selected from a range of $61-100 \%$ were coupled with high-heat $\left(47^{\circ} \mathrm{C}\right)$ stimuli. Thus, the difference between expected and actual temperature increased linearly for the cue ranges of $1-40 \%$ and $61-100 \%$ between $0^{\circ} \mathrm{C}$ and $1.2^{\circ} \mathrm{C}$. There was no prediction error for cue ranges of $31-40 \%$ and $91-100 \%$.

In the third and fourth runs (Mismatched Level 2 condition), cue values were selected from a range of $1-40 \%$ and were again coupled with $45^{\circ} \mathrm{C}$ stimuli to introduce a low range of prediction errors; however, cue values ranging from 1 to $100 \%$ were paired with $47^{\circ} \mathrm{C}$ stimuli. The latter pairing introduced a higher range of prediction errors $\left(0-3.2^{\circ} \mathrm{C}\right)$ and there was no prediction error for cues between 91 and 100. In addition, in 22 trials, $45^{\circ} \mathrm{C}$ and $47^{\circ} \mathrm{C}$ stimulus intensities were paired with cues that stated "the incoming heat stimulus intensity is unknown" at baseline and interspersed between Level 1 and Level 2 mismatched trials (Fig. 1D). Visual presentation and behavioral data collection were controlled through Presentation software (Neurobehavioral Systems). The order of epochs was pseudorandomized, and a total of 42 epochs were presented in runs 2-4. Data for the baseline responses and first run were collected in the scanner in only 20 participants, and the scans were not used in the analysis.
fMRI data acquisition. Structural and functional data were collected on a 3.0 T MRI scanner (Discovery MR750, GE Healthcare) at the Halifax Infirmary site of the Queen Elizabeth II Health Sciences Centre. A T1weighted brain image (inversion recovery fast spoiled gradient recalled sequence (FPSGR)) was acquired using the following parameters: field of view $=224 \times 224 \mathrm{~mm}$; in-plane resolution $=1 \times 1 \mathrm{~mm}$; slice thickness $=1.0 \mathrm{~mm}$; TR $/ \mathrm{TE}=4.4 / 1.908 \mathrm{~ms}$; flip angle $=9^{\circ}$. $\mathrm{fMRI}$ data were acquired using a multiband EPI sequence: field of view $=216 \times 216 \mathrm{~mm}$; in-plane resolution $=3 \times 3 \mathrm{~mm}$; slice thickness $=3.0 \mathrm{~mm}$; TR $/ \mathrm{TE}=$ $950 / 30 \mathrm{~ms}$. Total number of volumes was 814 for the first matched functional scan and 624 for the three other functional runs. Four volumes of reverse-phase-encoded multiband EPI were also acquired before the resting-state scan. Each participant's head was fitted with foam padding to minimize head movement, and ear plugs were provided to reduce scanner noise. Participants were reminded to keep their head as still as possible before each scan.

Behavioral data analysis. Questionnaires were administered before the experimental trials. Pain catastrophizing was assessed with the pain catastrophizing scale (Sullivan et al., 1995), and mindfulness was assessed using the 39-item Five Facet Mindfulness Questionnaire (Baer et al., 2006). The Spielberger Trait Anxiety Inventory (STAI) was used to assess anxiety (Spielberger, 1983). For evaluating pain sensitivity, we used the method of limits to obtain three observations of thermal pain thresholds and thermal pain tolerance and used the averaged values in analyses (Defrin et al., 2006). Behavioral data were analyzed using SPSS 24 (IBM).

Behavioral analysis was based on a repeated-measures ANOVA with cue values as predictors and pain ratings as dependent variables. All effects that met the criteria of $p<0.05$ (two-tailed) were considered significant.

Neuroimaging data analysis. Field map-based distortion correction was performed for all functional datasets. Three mismatched fMRI datasets were preprocessed with the FMRIB Software Library (FSL; http:// www.fmrib.ox.ac.uk/fsl) and Analysis of Functional NeuroImages (AFNI; http://afni.nimh.nih.gov/afni), with the scripts provided by the 1000 Functional Connectomes Project (http://www.nitrc.org/projects/ fcon_1000; Biswal et al., 2010). Preprocessing using AFNI consisted of (1) discarding the first five EPI volumes to allow for signal equilibration, (2) motion correction of time series by aligning each volume to the mean image using Fourier interpolation, (3) skull stripping, and (4) getting an eighth image for use in registration. Next, preprocessing using FSL consisted of (1) spatial smoothing using a Gaussian kernel of full-width at half-maximum $=6 \mathrm{~mm}$, (2) grand-mean scaling of the voxel value, (3) temporal filtering $(0.005-0.3 \mathrm{~Hz}),(4)$ removing linear and quadratic trends, and (5) removing nine nuisance signals (global mean, CSF, white matter, six motion parameters) by regression.

For each participant, time series statistical analysis was performed using the FMRIB Improved Linear Model. For first-level analysis, the heat stimulation with $47^{\circ} \mathrm{C}$ duration for cue ranges: $1-20 \%, 21-40 \%$, 


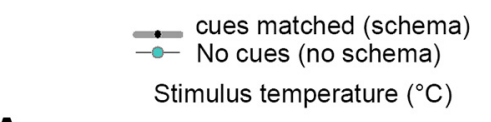

A

43.844 .244 .64545 .846 .246 .647

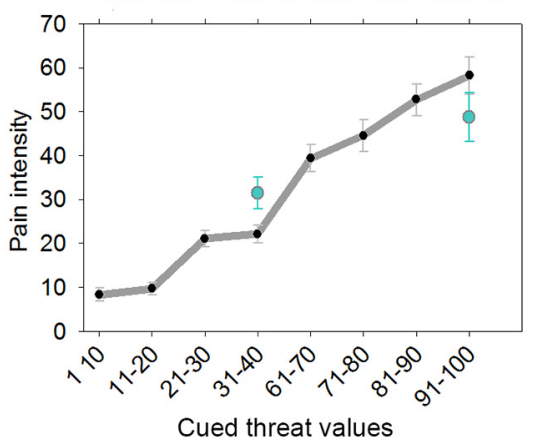

B

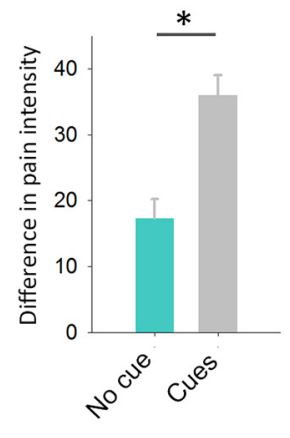

Figure 2. The linear schema altered pain perception for cued relative to uncued heat stimuli. $A$, Mean pain ratings sorted based on cued threat values demonstrate that the values linearized the evaluations of pain. Cyan dots represent mean pain rating observed at baseline in response to pseudorandom repetitions of uncued stimuli of $45^{\circ} \mathrm{C}$ and $47^{\circ} \mathrm{C}$. B, Difference in pain intensity between responses to $45^{\circ} \mathrm{C}$ and $47^{\circ} \mathrm{C}$ stimuli presented without cued (cyan bar) or with cued threat values (31-40 and 91-100, gray bar). Data are expressed as the mean \pm SEM. ${ }^{*} p<0.05$.

$61-80 \%$, and $91-100 \%(1-20 \%)$ was convolved with a canonical hemodynamic response function (gamma function: mean \pm SD lag, $6 \pm 3 \mathrm{~s}$ ). Temporal derivatives of the events were added to the model. Each participant's contrast images of parameter estimates were then transformed to standard space $\left(2 \times 2 \times 2 \mathrm{~mm}^{3}\right.$ resolution; 152 template, Montreal Neurological Institute) using the FMRIB Linear Image Registration Tool. First, functional images were aligned to the structural image with 6 degrees of freedom affine transformation. Then, the structural image was aligned to standard space with a 12 degree of freedom affine transformation. Finally, the resulting transformation matrix was applied to each participant's statistical data. A second-level analysis was performed to combine all three functional runs for individual participants using a fixed-effects model. Higher-level analysis was performed using the FMRIB Local Analysis of Mixed Effects (FLAME 1). To increase the number of observations, epochs of $47^{\circ} \mathrm{C}$ heat stimuli paired with a cue range of 1-20 (prediction error of $0^{\circ} \mathrm{C}$ ) and $47^{\circ} \mathrm{C}$ paired with a cue range of 91-100 (prediction error range of $2.8-3.2^{\circ} \mathrm{C}$ ) were contrasted with each other. Multiple-comparison corrections were performed using Gaussian random field theory (minimum $Z>2.3$; cluster level, $p<0.05$ ).

\section{Results}

\section{Pain perception is influenced by threat predictions}

As expected, baseline ratings for two different heat temperatures observed before schema-cue induction were significantly different $\left(31.51 \pm 3.6 \mathrm{SEM}\right.$ for $45^{\circ} \mathrm{C}$ and $48.78 \pm 5.53 \mathrm{SEM}$ for $47^{\circ} \mathrm{C}$; difference $=17.27$ on NRS, $T=391, p=0.016, t$ test rank sum). In the subsequent matched condition, where the cues linearly predicted the stimulus intensity, the response curve of pain ratings showed a linear pattern, confirming that participants correctly detected the implicit rule (Fig. $2 A$ ).

The extent to which pain perceived from $45^{\circ} \mathrm{C}$ differed from $47^{\circ} \mathrm{C}$ was significantly higher for the cued stimuli (paired with 31-40 and 91-100, respectively) relative to uncued baseline responses $(T=456, p<0.001$; Fig. $2 B)$, demonstrating that pain perception was altered to be more aligned with the linear increase in cued threat values than simple, bottom-up temperature effects.

Sensory evidence had lower contribution to pain perception relative to threat predictions from the linear schema

To challenge the schema, in the first set of stimuli, a low range of prediction errors was introduced (Mismatched Level 1). The
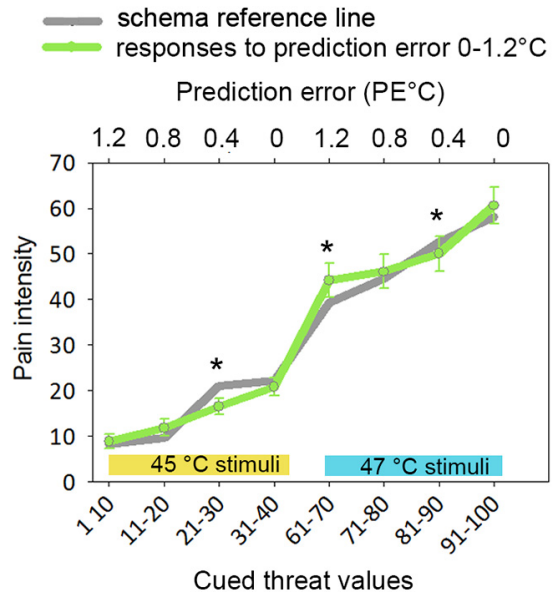

Figure 3. Effects of prediction errors in schema on pain responses for Mismatched Level 1 The pain response curve for mismatched cue values and heat stimuli. Cues ranging between 1 and 40 were paired with $45^{\circ} \mathrm{C}$, and those ranging between 61 and 100 were paired with $47^{\circ} \mathrm{C}$, resulting in a range of $P E s$ between $0^{\circ} \mathrm{C}$ and $1.2^{\circ} \mathrm{C}$. The gray line represents the response curve for the matched condition. Data are expressed as the mean \pm SEM. ${ }^{*} p<0.05$.

stimulus temperature diverged from the predicted threat value at a range from $0^{\circ} \mathrm{C}$ to $1.2^{\circ} \mathrm{C}$. The extent to which pain ratings were updated from the matched condition by the prediction errors was small but significant. Two-way repeated-measure ANOVA (matched vs unmatched conditions $\times$ cued threats at eight levels) revealed a significant main effect (Matched vs Unmatched Level 1 temperature $\times$ linear cued threat: $\left.F_{(1,285)}=8.442, p<0.001\right)$. Although the effect of cues was significant (1-10, 11-20, 21-30, $\left.31-40,61-70,71-80,91-100 ; F_{(7,287)}=141.86, p<0.001\right)$, the effect of matched versus unmatched temperature was not significant $\left(F_{(7,41)}=0.085, p=0.771\right)$. Post hoc analysis showed that the mean pain intensity was significantly updated for some but not all cue conditions (Fig. 3).

For Mismatched Level 2, cued threats ranged between 1 and 100 , but heat stimuli were held at a single intensity of $47^{\circ} \mathrm{C}$ (Figs. 1, 4A; Mismatched Condition Level 2). Two-way repeatedmeasure ANOVA (matched vs unmatched conditions $X$ cued threats at eight levels) revealed a significant main effect (matched vs unmatched temperature $\times$ linear cued threat: $F_{(1,285)}=17.62$, $p<0.001)$. The effect of cues $(1-10,11-20,21-30,31-40,61-70$, $\left.71-80,81-90,91-100 ; F_{(7,287)}=124.44, p<0.001\right)$ and the effect of matched versus unmatched temperature were also significant $\left(F_{(7,41)}=18.43, p<0.001\right)$. Post hoc analysis showed that the mean pain intensity was significantly greater for five of eight cue ranges than the matched condition (Fig. 4A).

This effect of bias in pain perception was evaluated as the difference in perceived pain with increase in stimulus strength between the $0 \mathrm{PE}$ and max PE within the Mismatched Level 2 condition, which showed a significant difference $(T=1160.5$, $p<0.001$, Mann-Whitney $U$ test, Cohen's $d=1.608$; Fig. $4 B$ ). In addition, update in pain ratings was quantified by measuring the change in bias with increase in PEs between the unmatched and matched conditions (Fig. 4C). This was quantified by first estimating the difference in pain intensity at different levels of $\mathrm{PE}$ relative to no $\mathrm{PE}$ within the mismatched condition and then calculating the percentage change of these values relative to differences for the same cues in the matched condition. The linear increase in magnitude of prediction errors resulted in an incrementing update and reduction in bias (Fig. 4C). The maximum value of update was at a mean of $27 \pm 3.15$ SEM. 
A

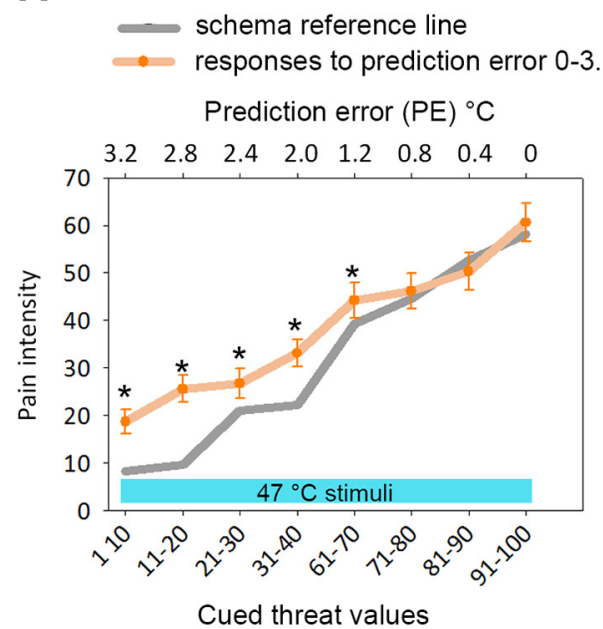

B

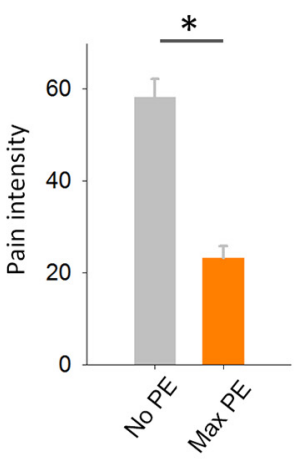

C

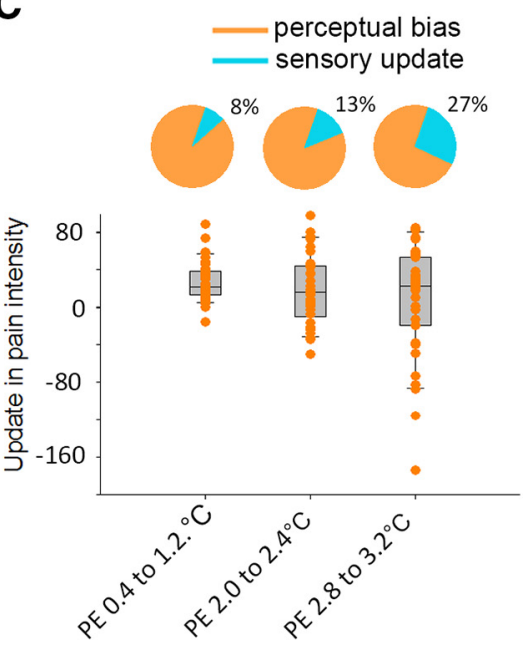

Figure 4. Effects of prediction errors in threat schema on pain responses. $A$, The pain response curve for mismatched cued threat and heat stimuli. Cued threat values were selected from a range of $1-100$, but the preceding stimulus was consistently at $47^{\circ} \mathrm{C}$, resulting in a range of prediction errors $\left(0-3.2^{\circ} \mathrm{C}\right)$. The gray line represents response curve for matched condition. $\boldsymbol{B}$, The pain ratings for the $\mathrm{PEE}$ versus the max PE were significantly different. The difference demonstrates a strong effect of cued threats on pain intensity for the same stimulus intensity and is taken to represent perceptual bias. Data are expressed as the mean \pm SEM. ${ }^{*} p<0.05$. C, The reduction in influence of cued threats on pain intensity by the change in sensory evidence is taken to represent an update. Pie charts show the mean percentage of ratings that were updated with change in sensory update relative to the expectation-induced bias in pain ratings. Scatter plots overlaid on box plots show an update in pain ratings for different levels of PEs.

The brain systems that mediate perceptual bias showed graded change in activity corresponding to the level of cued threat and prediction error

When the heat stimulus was held at $47^{\circ} \mathrm{C}$, several brain regions modulated their activity in relation with changes in cue temperature values. fMRI responses to heat stimuli were significantly different when predicted temperature was low (1-20) and PE from heat stimulus was at its $\max \left(2.8-3.2^{\circ} \mathrm{C}\right)$ relative to high cued threat (91-100) and no PE (FLAME 1, corrected at $p<0.05$; Fig. 5).

The regions that showed reduced activation in response to heat stimuli when cued threat was low and PE was at the maximum range were observed primarily in sensory areas, such as the secondary somatosensory cortex (S2), dorsal posterior insula (dpINS), middle insula (mINS; data not shown), putamen, nucleus cuneiformis/periaqueductal gray area (NCF/PAG) extending into the amygdala, and hippocampus in the left hemisphere (Fig. 5A; Table 1). In contrast, the brain regions that showed increased activation were mostly those that are related to cognitive processes: left dorsolateral prefrontal cortex (dlPFC), left inferior parietal lobule/supramarginal gyrus (SMG), left lateral orbitofrontal cortex (lOFC), ventrolateral prefrontal cortex (vlPFC), and right ventromedial prefrontal cortex (vmPFC; Fig. $5 B$; Table 1). The observed effects in cognitive and sensory systems may reflect the top-down cognitive influence of prior information on pain perception and the regions important for perceptual processing of pain and learning from prediction errors in nociceptive inputs.

Next, for each identified region in the cognitive and sensory network, brain activation (parameter estimates) was extracted for events at four levels of prediction errors $\left(0^{\circ} \mathrm{C}, 0.8-1.2^{\circ} \mathrm{C}, 2.0-\right.$ $2.4^{\circ} \mathrm{C}$, and $2.8-3.2^{\circ} \mathrm{C}$ ). When responses were ranked based on ascending cue values, most regions showed a graded response that corresponded with predictive values even though the stimulus was held at a single temperature of $47^{\circ} \mathrm{C}$. All regions tracked the linear schema, and this effect was most prominent for left putamen and left dlPFC as shown in Figure 5, $C$ and $D$.
Effect of linear schema on pain responses to uncued (schemafree) stimuli presented during mismatched trials

One-way repeated-measure ANOVA showed a significant main effect on pain responses of cued versus uncued conditions (ANOVA on ranks, $H=37.89, p<0.001$ ) for the $47^{\circ} \mathrm{C}$ stimuli (Fig. 6A). Thus, pain responses to uncued stimuli were different from cued stimuli with max PE (difference of ranks $=1403, q=$ $5.92, p<0.05,47^{\circ} \mathrm{C}$ ) but not different from cued stimuli with no $\mathrm{PE}$ (difference of ranks $=605, q=2.55, p>0.05,47^{\circ} \mathrm{C}$ ). Thus, $47^{\circ} \mathrm{C}$ continued to be identified as more painful when there were no explicit cued threats predicting the stimulus strength, and this evaluation relied on bottom-up nociceptive signals received directly from the stimulus. But when the same intensity was paired with cues that signaled low threat, the evaluated pain was prominently reduced.

Furthermore, the contrast between uncued stimulus intensities became linear during the mismatched condition. The difference in pain ratings between the uncued stimuli $\left(45^{\circ} \mathrm{C}\right.$ vs $\left.47^{\circ} \mathrm{C}\right)$ was significantly higher $(T=528, p=0.008)$ during the mismatched trials (mean \pm SEM, $27.61 \pm 2.45$ ) relative to baseline (mean \pm SEM, $17.267 \pm 2.93$ ). Thus, responses to cued threat values and to uncued stimuli were both affected by the linear schema (figure not shown).

\section{Schema-related versus schema-free heat pain stimuli activate different brain networks}

Next, we identified regions that mediate cued threat-based pain perception relative to regions that mediate pain perception based primarily on the evoking stimulus. Activations in response to $47^{\circ} \mathrm{C}$ heat stimuli paired with matched cued threat values (91$100 ; 0 \mathrm{PE}$ ) were contrasted with $47^{\circ} \mathrm{C}$ heat stimuli presented without cue values (whole-brain-corrected $p<0.05$, FLAME 1). Activations in response to cued heat stimuli were significantly greater in the right dlPFC, right and left frontopolar PFC (Brodmann area 10), and right dorsal anterior cingulate cortex (ACC; Fig. $6 \mathrm{~B}$; Table 2). In the absence of cued threat values, the $47^{\circ} \mathrm{C}$ 
A

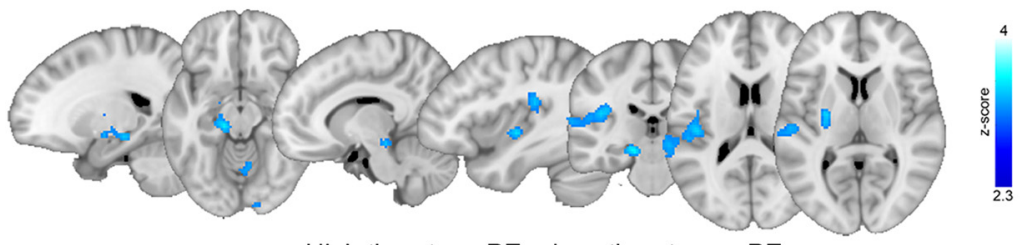

High threat, no PE > Low threat, max PE

B

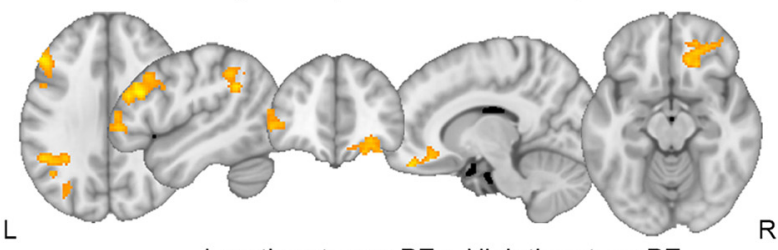

Low threat, max PE > High threat, no PE

Corrected FLAME1

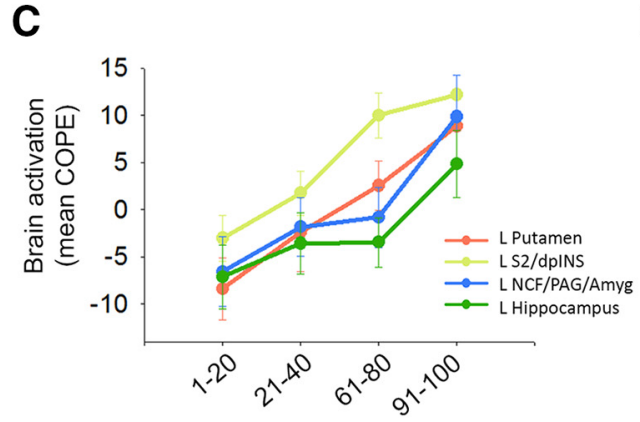

Cued threat values at $47^{\circ} \mathrm{C}$
D

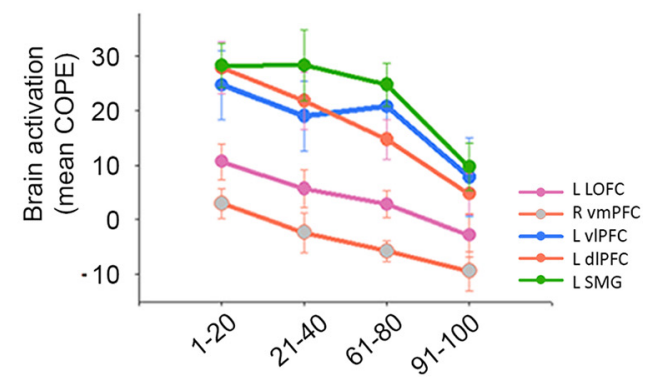

Cued threat values at $47^{\circ} \mathrm{C}$

Figure 5. Cognitive, striatal, and sensory brain regions mediate perceptual bias. $A, B$, Contrasts between high cued threat (91-100) at no PE relative to low cued threat (1-20) and high PE $\left(2.8-3.2^{\circ} \mathrm{C}\right)$. Heat stimulus intensity was constant at $47^{\circ} \mathrm{C} . \boldsymbol{C}, \boldsymbol{D}$, Identified regions show graded change in relation to changes in cued threats. Parameter estimates were extracted from a $6 \mathrm{~mm}$ sphere around the peak voxels of activation at different levels of cued threats/PEs. A $3 \mathrm{~mm}$ sphere was used for PAG.

Table 1. Comparison of brain activation during heat pain stimuli $\left(47^{\circ} \mathrm{C}\right.$ ) paired with 1-20 (max PE) and $91-100$ cue values (no PE)

\begin{tabular}{|c|c|c|c|c|}
\hline Contrast & Brain region & Peak coordinate $(x, y, z)$ & Zvalue & Number of voxels \\
\hline \multirow[t]{5}{*}{$\operatorname{Max} P E(1-20)>$ no PE (91-100) } & Left lateral orbitofrontal cortex & $-38,36,-8$ & 3.02 & 40 \\
\hline & Left ventrolateral prefrontal cortex & $-50,44,-2$ & 3.24 & 202 \\
\hline & Left dorsolateral prefrontal cortex & $-50,30,28$ & 4.04 & 428 \\
\hline & Left supramarginal gyrus & $-32,-50,34$ & 3.77 & 977 \\
\hline & Right ventromedial prefrontal cortex/medial orbitofrontal cortex & $18,46,-20$ & 3.92 & 469 \\
\hline \multirow[t]{5}{*}{ No $P E(91-100)>\max P E(1-20)$} & Left secondary somatosensory cortex/dorsal posterior insula & $-42,-24,18$ & 4.00 & 527 \\
\hline & Left putamen & $-34,-8,-2$ & 3.95 & 170 \\
\hline & Left parahippocampal gyrus & $-18,-28,-12$ & 3.81 & 32 \\
\hline & Left nucleus cuneiformis/amygdala & $-12,-28,-14$ & 4.09 & 67 \\
\hline & Right lingual gyrus & $8,-78,0$ & 3.91 & 506 \\
\hline
\end{tabular}

stimulus evoked significantly greater activity in the left frontal, and visual cortex (Fig. 6C, Table 2).

The right and left nucleus accumbens, intraparietal lobule, dorsomedial PFC, OFC, and dlPFC activated when the level of threat was low relative to absence of cue values (i.e., 1-20 vs no cues; Fig. $6 D$ ). In the opposite contrast (Fig. $6 E$ ), absence of cues was related to greater activations in sensory regions, including parietal cortex, visual cortex, and somatosensory area 1 (leg region ipsilateral to heat stimulus).

\section{Individual differences in perceptual bias are linked with} striatal circuitry, catastrophizing, and sensory awareness To understand why some individuals are more affected by threat predictions, we quantified bias for individuals as the difference in pain ratings evoked by the $47^{\circ} \mathrm{C}$ stimuli when they were paired with cue values of 91-100 (no PE) compared with 1-20 ( $\max P E)$.
The mean extent of bias measured as the absolute difference between the lowest $\left(0^{\circ} \mathrm{C}\right)$ and highest $\left(2.8-3.2^{\circ} \mathrm{C}\right)$ prediction error was $37.1 \pm 3.6$ (mean \pm SEM), and the range was between 3.3 and 70 (mean absolute difference in NRS). On investigating factors that mediate individual differences, bias was observed to be significantly higher in individuals who reported lower mindfulness on the Five Facet Mindfulness Questionnaire (Fig. 7). Of the 10 brain regions that showed a significant change in activation to heat stimuli between the 0 versus max $\mathrm{PE}$ condition, the putamen was observed to significantly correlate positively with mindfulness scores (Fig. $7 B$ ) and negatively with bias (Fig. 7C).

To measure the role of threat sensitivity, self-reported pain catastrophization, anxiety and pain sensitivity measures (pain threshold and pain tolerance) were used to predict the variability in perceptual bias. A higher score on pain catastrophizing was 


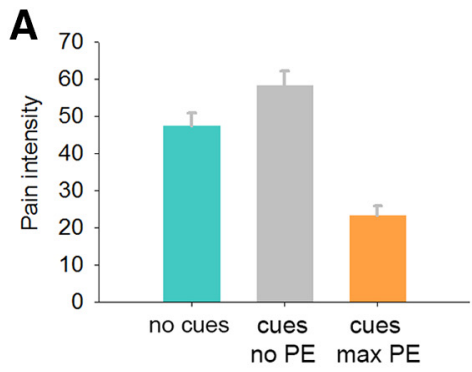

B

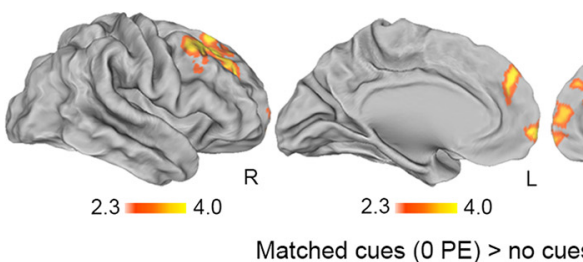

Matched cues $(\mathrm{OPE})>$ no cues
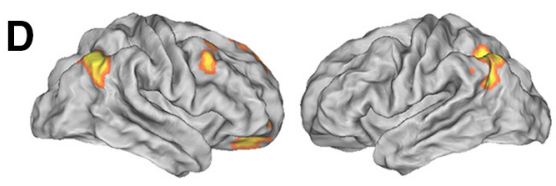

E

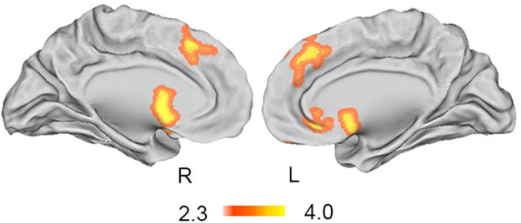

Unmatched cues at max $P E>$ no cues
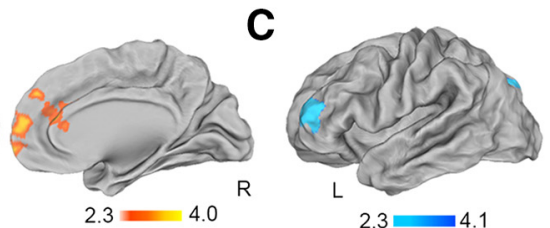

No cues $>$ matched cues (O PE)
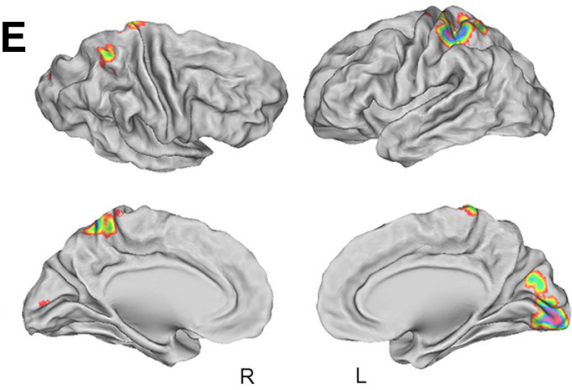

$2.3=4.0$

No cues > cues at max PE

Corrected FLAME 1

Figure 6. Comparisons between un-cued (schema-free) versus cued stimuli (schema-related) responses to heat stimuli. $A$, The pain intensity evoked by $47^{\circ} \mathrm{C} s$ timuli was similar between the schema-related (cued) and schema-free (uncued) condition when the cues had no PE, but the evaluations of cued stimuli were significantly lower when cued threat was low (max PE). $\boldsymbol{B}$, The contrast between $0 \mathrm{PE}$ and the no cue condition showed significantly more activations in the right dorsolateral, right and left dorsomedial and frontopolar prefrontal cortex, and right dorsal ACC than uncued heat stimuli. Note that heat stimulus intensity was $47^{\circ} \mathrm{C}$ in both conditions. C, The opposite contrast (no cue relative to cued condition) showed activation in the left dorsolateral prefrontal cortex and visual cortex. $\boldsymbol{D}$, Heat stimuli paired with cue values of $1-20$ activated several regions when contrasted against uncued heat stimuli: right and left nucleus accumbens, intraparietal lobule, subgenual ACC, dorsomedial PFC, orbitofrontal cortex, and dorsolateral PFC. Heat stimulus intensity was $47^{\circ} \mathrm{C}$ in both conditions, and the cue values predicted the stimulus intensity to be $2.8-3.2^{\circ} \mathrm{Cless}$ than the actual stimulus intensity (i.e., max PE). $\boldsymbol{E}$, The opposite contrast showed activation in the sensory regions, including the parietal cortex, visual cortex, and somatosensory area 1 (leg region ipsilateral to heat stimulus).

Table 2. Comparison of brain activation during predictable (cued) and unpredictable (uncued) heat pain stimuli $\left(47^{\circ} \mathrm{C}\right.$ )

\begin{tabular}{|c|c|c|c|c|}
\hline Contrast & Brain region & Peak coordinate $(x, y, z)$ & $Z$ value & Number of voxels \\
\hline \multirow[t]{4}{*}{ Matched cues $(0 \mathrm{PE})>$ no cues } & Left medial prefrontal cortex & $-14,60,0$ & 3.62 & 422 \\
\hline & Right dorsolateral prefrontal cortex & $20,28,40$ & 4.15 & 869 \\
\hline & Right dorsal anterior cingulate cortex & $14,46,22$ & 3.44 & 344 \\
\hline & Right medial prefrontal cortex & $8,62,-4$ & 3.22 & 53 \\
\hline \multirow[t]{2}{*}{ No cues $>$ matched cues ( $0 \mathrm{PE}$ ) } & Left dorsolateral prefrontal cortex & $-38,52,74$ & 4.49 & 516 \\
\hline & Right cuneus & $6,-78,20$ & 3.61 & 392 \\
\hline \multirow[t]{7}{*}{ Unmatched cues at max PE $>$ no cues } & Left inferior/superior parietal lobule & $-32,-52,34$ & 4.24 & 962 \\
\hline & Left and right dorsomedial prefrontal cortex & $-6,22,42$ & 3.89 & 445 \\
\hline & Left nucleus accumbens/caudate & $-6,8,-6$ & 3.40 & 146 \\
\hline & Right dorsolateral prefrontal cortex & $46,16,44$ & 4.28 & 533 \\
\hline & Right inferior/superior parietal lobule & $44,-56,42$ & 4.57 & 1190 \\
\hline & Right nucleus accumbens/caudate & $12,10,-4$ & 3.57 & 272 \\
\hline & Right orbitofrontal cortex & $20,44,-18$ & 4.24 & 556 \\
\hline \multirow[t]{3}{*}{ No cues $>$ unmatched cues at max PE } & Left and right postcentral gyrus & $0,-38,66$ & 3.8 & 747 \\
\hline & Left postcentral gyrus & $-40,-30,50$ & 3.81 & 661 \\
\hline & Right occipital pole & $12,-94,-12$ & 3.32 & 615 \\
\hline
\end{tabular}

linked with stronger bias toward cued threat values $(r=0.410$, $p=0.008$, Fig. 8A). Moreover, higher perceptual bias significantly predicted lower pain tolerance $(r=-0.372, p=0.0169$, Fig. $8 B$ ) but did not predict pain thresholds $(r=0.201, p=$ $0.207)$. Other tested factors that were not significantly related were age, sex, and anxiety scores $(p>0.05)$. For this analysis, we measured the difference in responses of accumbens and vmPFC between states of no PE and max PE. The accumbens showed a small but significant increase and the vmPFC decreased in responsiveness (Fig. 5) with increase in PE. The extent of change in activations of the vmPFC and accumbens were observed to significantly correlate with pain catastrophizing (Fig. $8 C$ and $D$ ). Perceptual bias was significantly linked with change in accumbens $(r=0.363, p=0.025)$ and marginally linked with change in vmPFC $(r=0.285, p=0.088)$ responses to $\mathrm{PE}$.

\section{Discussion}

This study demonstrates that threat predictions generated from cognitive schemas continue to influence pain perception despite increasing prediction errors arising from the sensory evidence in pain pathways. Perceptual bias was significantly reduced with every increase in prediction error but could not be fully corrected, even when the prediction error was at its highest (effect size for difference in no PE and max PE = 1.608). Overall, these observations extend and complement model-based studies in showing 
A

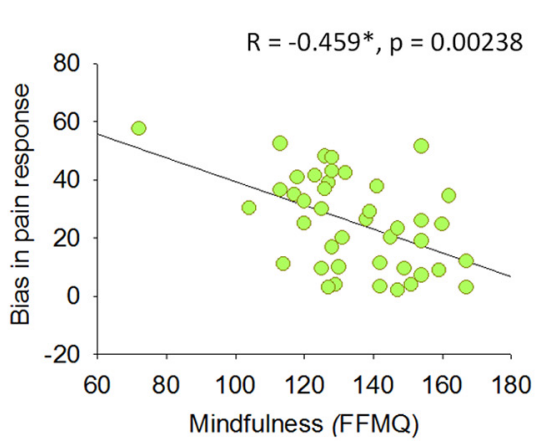

B

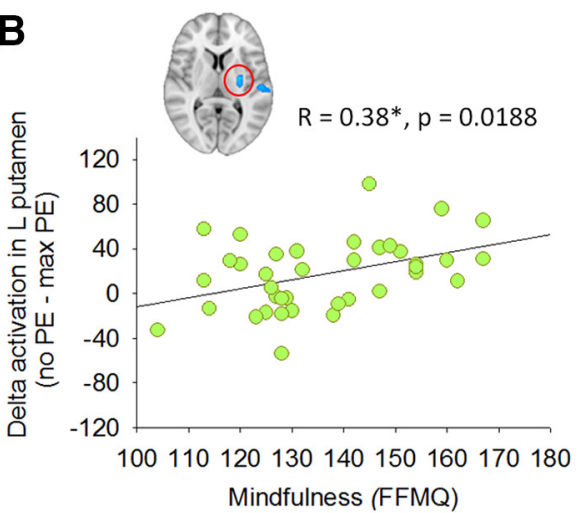

C

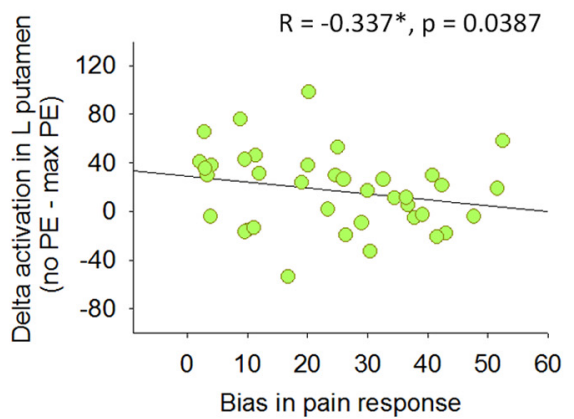

Figure 7. Low mindful awareness and reduced putamen responsiveness to cues are involved in perceptual bias. $A$, Participants with a higher level of perceptual bias (difference in pain ratings evoked in response to $47^{\circ} \mathrm{C}$ paired with cue values of $91-100$ and $1-20$ ) reported lower scores on mindful awareness measured with the Five Facet Mindfulness Questionnaire (FFMQ). $\boldsymbol{B}$, At the group level, putamen activation was decreased by the low cue values $(1-20 ;$ Fig. 5$)$, but at the individual level, the putamen response to heat decreased relatively less in individuals who scored lower on the mindfulness questionnaire. $\boldsymbol{C}$, This effect was also observed in individuals who reported greater perceptual bias.

A
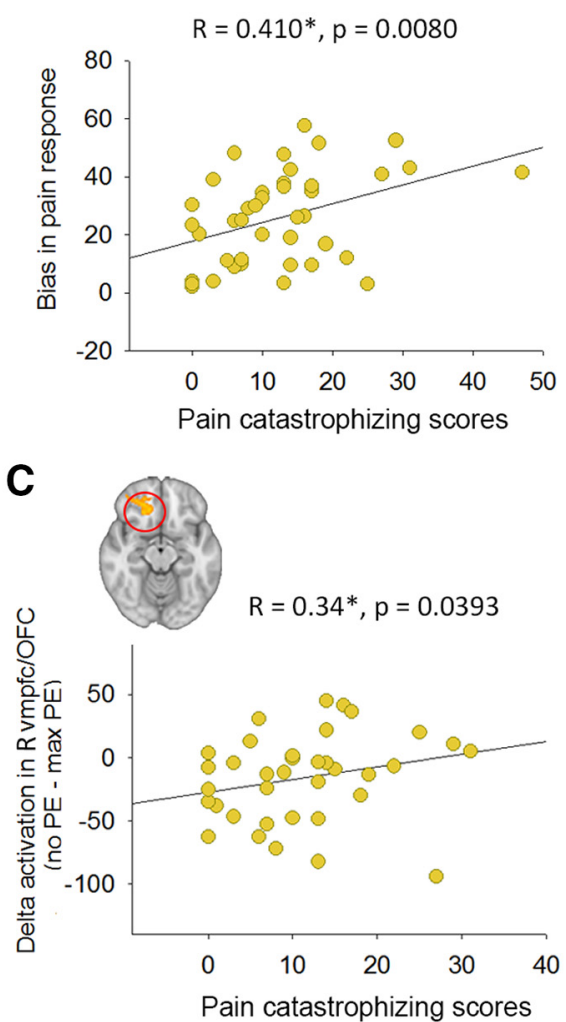

B

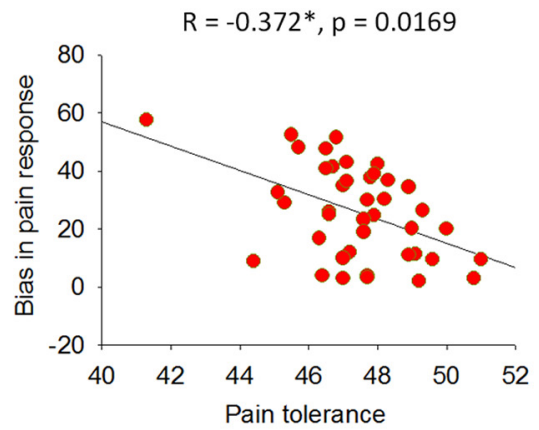

D

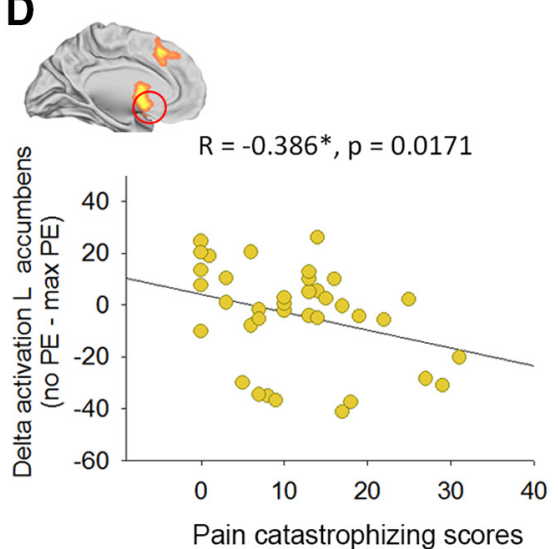

Figure 8. High catastrophizing and reduced activity of vmPFC are involved in perceptual bias. $\boldsymbol{A}, \boldsymbol{B}$, Higher perceptual bias toward cued threats was related to higher pain catastrophizing $(\boldsymbol{A})$ and lower heat pain tolerance $(\boldsymbol{B})$. $\boldsymbol{C}, \boldsymbol{D}$, Reward circuitry responses to PEs were affected by pain catastrophizing. $\boldsymbol{C}$, Decrease in vmPFC responses with increase in PE was greater in individuals with high pain catastrophizing. $\boldsymbol{D}$, Increase in accumbens responses with increase in PE was lower in individuals with high pain catastrophizing.

a mechanistic role of predictions in influencing pain perception (Büchel et al., 2014; Anchisi and Zanon, 2015).

Previous studies have investigated whether expectations formed by conditioning can modulate pain with relatively simple tasks and pavlovian cues (Atlas and Wager, 2012; Roy et al., 2014; Wiech et al., 2014; Geuter et al., 2017; Tinnermann et al., 2017; Wang et al., 2018). This study complements previous findings and extends them by showing that an increase in prediction errors is ignored in uncertain conditions that require difficult mental transformations of information (Sharot et al., 2007; Hohwy, 2017; Sarafyazd and Jazayeri, 2019). For complex tasks, with varied inputs, bias acts as a compensation mechanism for dealing with uncertainty. This study offers empirical evidence for the influence of learning and inference on pain intensity and offers new insights into the nature of pain as a signal for detecting and evaluating threats. The role of threat detection in biasing pain perception has been suggested, and there is a need to re-evaluate classical assumptions of pain as a bottom-up system for representing properties of noxious stimuli (Craig, 2003; Hashmi, 2018). Through this research, a new understanding of pain is emerging to review the role of pain as a learning signal that reinforces aversions to threats in addition to signaling actual or potential tissue damage (Davis et al., 2015; Wiech, 2016; Apkarian, 2019; Seymour, 2019). Pain enables an important ability in organisms to learn contingencies related to punishing experiences and adaptively guides actions on safely exploring the environment and preventing harm (Daw and Doya, 2006; Donoso et al., 2014; LeDoux and Daw, 2018).

Changes in the levels of cued threats altered activity in cognitive and sensory/ subcortical networks in a graded and opposite manner, and this observation underscores a role of these respective topdown and bottom-up systems in biasing pain perception. The striatal and prefrontal circuitry are centrally involved in adapting behavior to changing contingencies (Diuk et al., 2013; Donoso et al., 2014; Wilson et al., 2014; Niv et al., 2015). These observations direct us to the known role of these regions in controlling attention (Leong et al., 2017), maintaining task set in working memory (Momennejad et al., 2018; Rouhani et al., 2018), evaluating choices (Wang et al., 2018; Ardid et al., 2019), and confidence (Ma and Jazayeri, 2014) while monitoring sensory evidence aris- 
ing from the bottom-up pathways of other sensory modalities, such as visual information (Pessoa et al., 2003). The top-down influence on the perceptual experience of pain observed in this study defines the function of these top-down systems in predicting and evaluating physical threats (Wager et al., 2004; Tinnermann et al., 2017; Seymour, 2019) and signifies that these systems engage in processing cues from all sensory modalities to enable threat detection to adjust pain experience and behavior (LeDoux and Daw, 2018). Moreover, the graded effect of cues on activation in these regions demonstrates the extent to which responses of these regions can become entrained by changing magnitudes of predicted threat. These sets of systems are reported to maintain the status quo putatively by biasing attention and working memory toward predictions and exploiting previously learned strategy of the matched condition (Sarafyazd and Jazayeri, 2019).

In contrast, regions that are widely implicated for their role in pain perception, threat detection, and fear conditioning, such as $\mathrm{S} 2$, posterior insula, and $\mathrm{PAG} / \mathrm{NCF} /$ amygdala (Leknes et al., 2013; Roy et al., 2014; Davis et al., 2015; Sprenger et al., 2015; Berret et al., 2019), were responsive to increases in the extent of cued threat. The dorsal striatum and hippocampus also showed a similar pattern, demonstrating their role in the learning and processing of prediction errors, as has been suggested previously (Wang et al., 2018; Iigaya and O'Doherty, 2019; Pauli et al., 2019). Activations observed in the vmPFC region increased in relation to increase in prediction errors, further confirming its role in signaling expected value and aversive learning (Roy et al., 2012; Juechems et al., 2019). Relative to activation patterns in response to cued threats, the uncued stimuli activated sensory regions, including the leg area on the hemisphere contralateral to the site of heat stimulation, indicating increased monitoring of inputs from bottom-up pathways (Fardo et al., 2017).

Some important distinctions were observed from the variability between participants in the extent to which their pain reports could be biased by cued threats. This variability may be due to the extent to which they updated their pain responses based on changes in bottom-up sensory evidence. Another factor behind this variability is related to how participants varied in learning the rule during the first (matched) condition. For instance, high-bias individuals may have also been biased toward the cue values while learning the schema, resulting in more proficient learning of the implicit linear schema, or due to greater responsiveness to the encoded level of threat. Although the two factors (bias caused by adept learning vs. perseverance of learning) are entangled, the variability can be taken to represent how some individuals are more biased by the threat represented by the cues and hence show more reliance on schemas and less update when prediction errors are presented. Self-reported catastrophizing toward pain was higher and pain tolerance was lower in individuals who were influenced more by cued threat values than by the sensory evidence. The phenomenon was linked with responses of the vmPFC region and accumbens, where the former region showed less and the latter showed more change in responses with reduction in cued threat values in pain catastrophizers. This pattern of activity may be linked with mediating learning from prediction errors in schema or model-based predictions (Niv et al., 2012). The vmPFC region plays a central role in regulating negative emotions and representing value in decisions involving rewards and punishment under conditions of risk and ambiguities and is important for reversing contingencies (Roy et al., 2012; McNamee et al., 2013; Dunsmoor et al., 2019). This finding suggests that (1) individuals with higher sensitivity to predicted threats are more vulnerable to perceptual bias, and (2) encoding of learning signals in accumbens and vmPFC systems is influenced by sensitivity to threats, and this link may underpin a stronger influence of declarative and value-based systems (Wang et al., 2018; Seymour, 2019) on sensory decisions among threat-sensitive individuals.

Another related finding highlights the role of the dorsal striatum in mediating the link between low sensory awareness and higher perceptual bias toward predicted threats. Individuals with greater capacity for sustained attention toward bodily sensations were less biased by cued threats while evaluating the sensory evidence. Individual reports of mindfulness and perceptual bias were both linked with the extent to which the putamen increased its responsiveness to heat stimuli with increase in cued threats. In models of reinforcement learning, this region is implicated in gauging prediction errors in learned associations that underpin habitual actions (Doll et al., 2015; Gillan et al., 2015; Otto et al., 2015; Wang et al., 2018). This region is hypothesized to be a central player in the system that encodes offline computations of aversive learning and decisions that are more habit-like and less explicit or cognitive in their nature (Wang et al., 2018). In conditions of high uncertainty, this alternative system is suggested to take over as a default system when the need for updating the model is unclear. Together with the presented findings, mindfulness and its link with signaling of prediction errors within the putamen (dorsal striatum) may suggest that mindfulness increases reliance on these alternative systems (Wang et al., 2018) in shaping sensory decisions so that they are aligned more on sensory inputs rather than on overt or conceptual threat predictions, but this assumption needs further verification.

These findings carry clinical, societal, and scientific implications. First, if we assume that perceptions accurately map to physical events, the systems that transmit and represent external events as internal percepts are prone to nonlinearities so that our subjective experiences are not absolute one-to-one reconstructions of objective details of noxious stimuli. Such intransitive disparities are especially observed for pain experiences where the experienced intensity of pain perception changes with context. Clinically and in pain psychophysics, many interesting departures from theories of bottom-up or labeled-line pain processing have been noted (Wiech, 2016; Reddan and Wager, 2018; Apkarian, 2019; Seymour, 2019). An even more significant consequence of this disparity between object and participant are the chronic pain conditions that afflict a large percentage of the human population (Davis and Seminowicz, 2017; Apkarian, 2019; Tracey et al., 2019). These individuals suffer from pains that often do not correspond to external causes or symptoms of tissue damage or pathology (Davis et al., 2017). When taken together with studies that measure changes in pain response with context, the take-home implication is that pain is perceived differently in each individual due to the threat level individuals detect from the environmental context or prior experiences. The bias toward context in evaluating pain is also influenced by threat sensitivity and lower bodily awareness. From this vantage point, the subjective and incorrigible aspects of pain experience are less paradoxical than have been previously surmised.

Overall, our findings demonstrate that pain perception is a combination of predicted threat and sensory inputs in pain pathways. The bias exerted by schemas is reduced when prediction errors increase, but the update is partial and even less prominent in individuals who are innately fearful of pain and more prominent in individuals with higher mindful awareness. The brain 
systems that mediate these effects are those involved in cognitive processing and aversive learning.

\section{References}

Anchisi D, Zanon M (2015) A Bayesian perspective on sensory and cognitive integration in pain perception and placebo analgesia. PLoS One 10:e0117270.

Apkarian AV (2019) Definitions of nociception, pain, and chronic pain with implications regarding science and society. Neurosci Lett 702:1-2.

Ardid S, Sherfey JS, McCarthy MM, Hass J, Pittman-Polletta BR, Kopell N (2019) Biased competition in the absence of input bias revealed through corticostriatal computation. Proc Natl Acad Sci U S A 116:8564-8569.

Ashar YK, Chang LJ, Wager TD (2017) Brain mechanisms of the placebo effect: an affective appraisal account. Annu Rev Clin Psychol 13:73-98.

Atlas LY, Wager TD (2012) How expectations shape pain. Neurosci Lett 520:140-148.

Baer RA, Smith GT, Hopkins J, Krietemeyer J, Toney L (2006) Using selfreport assessment methods to explore facets of mindfulness. Assessment 13:27-45.

Baliki MN, Geha PY, Fields HL, Apkarian AV (2010) Predicting value of pain and analgesia: nucleus accumbens response to noxious stimuli changes in the presence of chronic pain. Neuron 66:149-160.

Berret E, Kintscher M, Palchaudhuri S, Tang W, Osypenko D, Kochubey O, Schneggenburger R (2019) Insular cortex processes aversive somatosensory information and is crucial for threat learning. Science 364:eaaw0474.

Biswal BB, Mennes M, Zuo XN, Gohel S, Kelly C, Smith SM, Beckmann CF, Adelstein JS, Buckner RL, Colcombe S, Dogonowski AM, Ernst M, Fair D, Hampson M, Hoptman MJ, Hyde JS, Kiviniemi VJ, Kötter R, Li SJ, Lin $\mathrm{CP}$, et al. (2010) Toward discovery science of human brain function. Proc Natl Acad Sci U S A 107:4734-4739.

Boichat C, Eccleston C, Keogh E (2018) The tripartite structure of painrelated affect: a confirmatory factor analysis. Psychol Health Med 23: 1211-1222.

Büchel C, Geuter S, Sprenger C, Eippert F (2014) Placebo analgesia: a predictive coding perspective. Neuron 81:1223-1239.

Craig AD (2003) Interoception: the sense of the physiological condition of the body. Curr Opin Neurobiol 13:500-505.

Davis KD, Seminowicz DA (2017) Insights for clinicians from brain imaging studies of pain. Clin J Pain 33:291-294.

Davis KD, Kucyi A, Moayedi M (2015) The pain switch: an "ouch" detector. Pain 156:2164-2166.

Davis KD, Flor H, Greely HT, Iannetti GD, Mackey S, Ploner M, Pustilnik A, Tracey I, Treede RD, Wager TD (2017) Brain imaging tests for chronic pain: medical, legal and ethical issues and recommendations. Nat Rev Neurol 13:624-638.

Daw ND, Doya K (2006) The computational neurobiology of learning and reward. Curr Opin Neurobiol 16:199-204.

Defrin R, Shachal-Shiffer M, Hadgadg M, Peretz C (2006) Quantitative somatosensory testing of warm and heat-pain thresholds: the effect of body region and testing method. Clin J Pain 22:130-136.

Diuk C, Tsai K, Wallis J, Botvinick M, Niv Y (2013) Hierarchical learning induces two simultaneous, but separable, prediction errors in human basal ganglia. J Neurosci 33:5797-5805.

Doll BB, Shohamy D, Daw ND (2015) Multiple memory systems as substrates for multiple decision systems. Neurobiol Learn Mem 117:4-13.

Donoso M, Collins AG, Koechlin E (2014) Human cognition. foundations of human reasoning in the prefrontal cortex. Science 344:1481-1486.

Dunsmoor JE, Kroes MCW, Li J, Daw ND, Simpson HB, Phelps EA (2019) Role of human ventromedial prefrontal cortex in learning and recall of enhanced extinction. J Neurosci 39:3264-3276.

Egner T, Summerfield C (2013) Grounding predictive coding models in empirical neuroscience research. Behav Brain Sci 36:210-211.

Elman I, Borsook D (2018) Threat response system: parallel brain processes in pain vis-à-vis fear and anxiety. Front Psychiatry 9:29.

Fardo F, Auksztulewicz R, Allen M, Dietz MJ, Roepstorff A, Friston KJ (2017) Expectation violation and attention to pain jointly modulate neural gain in somatosensory cortex. Neuroimage 153:109-121.

Feldman H, Friston KJ (2010) Attention, uncertainty, and free-energy. Front Hum Neurosci 4:215.

Friston K, Kiebel S (2009) Predictive coding under the free-energy principle. Philos Trans R Soc Lond B Biol Sci 364:1211-1221.
Garrett N, González-Garzón AM, Foulkes L, Levita L, Sharot T (2018) Updating beliefs under perceived threat. J Neurosci 38:7901-7911.

Geuter S, Boll S, Eippert F, Büchel C (2017) Functional dissociation of stimulus intensity encoding and predictive coding of pain in the insula. Elife 6:e24770.

Gillan CM, Otto AR, Phelps EA, Daw ND (2015) Model-based learning protects against forming habits. Cogn Affect Behav Neurosci 15:523-536.

Harrison R, Zeidan F, Kitsaras G, Ozcelik D, Salomons TV (2019) Trait mindfulness is associated with lower pain reactivity and connectivity of the default mode network. J Pain 20:645-654.

Hashmi JA (2018) Placebo effect: theory, mechanisms and teleological roots. Int Rev Neurobiol 139:233-253.

Hohwy J (2017) Priors in perception: top-down modulation, Bayesian perceptual learning rate, and prediction error minimization. Conscious Cogn 47:75-85.

Iigaya K, O’Doherty JP (2019) Hippocampus is what happens while you're busy making other plans. Neuron 102:517-519.

Juechems K, Balaguer J, Herce CastañónS, Ruz M, O’Reilly JX, Summerfield C (2019) A network for computing value equilibrium in the human medial prefrontal cortex. Neuron 101:977-987.e3.

Karos K, Williams ACC, Meulders A, Vlaeyen JWS (2018) Pain as a threat to the social self: a motivational account. Pain 159:1690-1695.

LeDoux J, Daw ND (2018) Surviving threats: neural circuit and computational implications of a new taxonomy of defensive behaviour. Nat Rev Neurosci 19:269-282.

Leknes S, Berna C, Lee MC, Snyder GD, Biele G, Tracey I (2013) The importance of context: when relative relief renders pain pleasant. Pain 154: 402-410.

Leong YC, Radulescu A, Daniel R, DeWoskin V, Niv Y (2017) Dynamic interaction between reinforcement learning and attention in multidimensional environments. Neuron 93:451-463.

Loeser JD, Treede RD (2008) The kyoto protocol of IASP basic pain terminology. Pain 137:473-477.

Ma WJ, Jazayeri M (2014) Neural coding of uncertainty and probability. Ann Rev Neurosci 37:205-220.

McNamee D, Rangel A, O'Doherty JP (2013) Category-dependent and category-independent goal-value codes in human ventromedial prefrontal cortex. Nat Neurosci 16:479-485.

Momennejad I, Otto AR, Daw ND, Norman KA (2018) Offline replay supports planning in human reinforcement learning. Elife 7:e32548.

Niv Y, Edlund JA, Dayan P, O’Doherty JP (2012) Neural prediction errors reveal a risk-sensitive reinforcement-learning process in the human brain. J Neurosci 32:551-562.

Niv Y, Daniel R, Geana A, Gershman SJ, Leong YC, Radulescu A, Wilson RC (2015) Reinforcement learning in multidimensional environments relies on attention mechanisms. J Neurosci 35:8145-8157.

O’Doherty JP, Cockburn J, Pauli WM (2017) Learning, reward, and decision making. Annu Rev Psychol 68:73-100.

Otto AR, Skatova A, Madlon-Kay S, Daw ND (2015) Cognitive control predicts use of model-based reinforcement learning. J Cogn Neurosci 27: 319-333.

Pauli WM, Gentile G, Collette S, Tyszka JM, O’Doherty JP (2019) Evidence for model-based encoding of pavlovian contingencies in the human brain. Nat Commun 10:1099.

Pessoa L, Kastner S, Ungerleider LG (2003) Neuroimaging studies of attention: from modulation of sensory processing to top-down control. J Neurosci 23:3990-3998.

Reddan MC, Wager TD (2018) Modeling pain using fMRI: from regions to biomarkers. Neurosci Bull 34:208-215.

Rigoli F, Friston KJ, Martinelli C, SelakovićM, Shergill SS, Dolan RJ (2016) A Bayesian model of context-sensitive value attribution. Elife 5:e16127.

Rouhani N, Norman KA, Niv Y (2018) Dissociable effects of surprising rewards on learning and memory. J Exp Psychol Learn Mem Cogn 44:1430 1443.

Roy M, Shohamy D, Wager TD (2012) Ventromedial prefrontal-subcortical systems and the generation of affective meaning. Trends Cogn Sci 16:147156.

Roy M, Shohamy D, Daw N, Jepma M, Wimmer GE, Wager TD (2014) Representation of aversive prediction errors in the human periaqueductal gray. Nat Neurosci 17:1607-1612.

Russek EM, Momennejad I, Botvinick MM, Gershman SJ, Daw ND (2017) 
Predictive representations can link model-based reinforcement learning to model-free mechanisms. PLoS Comput Biol 13:e1005768.

Sarafyazd M, Jazayeri M (2019) Hierarchical reasoning by neural circuits in the frontal cortex. Science 364:eaav8911.

Schenk LA, Sprenger C, Onat S, Colloca L, BüchelC (2017) Suppression of striatal prediction errors by the prefrontal cortex in placebo hypoalgesia. J Neurosci 37:9715-9723.

Seymour B (2019) Pain: a precision signal for reinforcement learning and control. Neuron 101:1029-1041.

Sharot T, Riccardi AM, Raio CM, Phelps EA (2007) Neural mechanisms mediating optimism bias. Nature 450:102-105.

Spielberger CD (1983) Manual for the State-Trait Anxiety Inventory STAI (form Y) ("self-evaluation questionnaire"). Palo Alto, CA: Consulting Psychologists.

Sprenger C, Finsterbusch J, BüchelC (2015) Spinal cord-midbrain functional connectivity is related to perceived pain intensity: a combined spino-cortical fMRI study. J Neurosci 35:4248-4257.

Sullivan MJL, Bishop SR, Pivik J (1995) The pain catastrophizing scale: development and validation. Psychol Assessment 7:524-532.

Tinnermann A, Geuter S, Sprenger C, Finsterbusch J, BüchelC (2017) Interactions between brain and spinal cord mediate value effects in nocebo hyperalgesia. Science 358:105-108.

Tracey I, Woolf CJ, Andrews NA (2019) Composite pain biomarker signatures for objective assessment and effective treatment. Neuron 101:783-800.
Wacongne C, Labyt E, van Wassenhove V, Bekinschtein T, Naccache L, Dehaene S (2011) Evidence for a hierarchy of predictions and prediction errors in human cortex. Proc Natl Acad Sci U S A 108:20754-20759.

Wager TD, Rilling JK, Smith EE, Sokolik A, Casey KL, Davidson RJ, Kosslyn SM, Rose RM, Cohen JD (2004) Placebo-induced changes in fMRI in the anticipation and experience of pain. Science 303:1162-1167.

Wang O, Lee SW, O’Doherty J, Seymour B, Yoshida W (2018) Modelbased and model-free pain avoidance learning. Brain Neurosci Adv 2:2398212818772964.

Weilnhammer VA, Stuke H, Sterzer P, Schmack K (2018) The neural correlates of hierarchical predictions for perceptual decisions. J Neurosci 38:5008-5021.

Wiech K (2016) Deconstructing the sensation of pain: the influence of cognitive processes on pain perception. Science 354:584-587.

Wiech K, Vandekerckhove J, Zaman J, Tuerlinckx F, Vlaeyen JW, Tracey I (2014) Influence of prior information on pain involves biased perceptual decision-making. Curr Biol 24:R679-R681.

Wilson RC, Takahashi YK, Schoenbaum G, Niv Y (2014) Orbitofrontal cortex as a cognitive map of task space. Neuron 81:267-279.

Zubieta JK (2010) Pain signal as threat and reward. Neuron 66:6-7.

Zunhammer M, Bingel U, Wager TD, Placebo Imaging Consortium (2018) Placebo effects on the neurologic pain signature: a meta-analysis of individual participant functional magnetic resonance imaging data. JAMA Neurol 75:1321-1330. 